\title{
Cinnamaldehyde Prevents Endothelial Dysfunction Induced by High Glucose by Activating Nrf2
}

\author{
Fang Wang ${ }^{a}$ Chunhua Pu Peng Zhou ${ }^{a}$ Peijian Wanga Dengpan Lianga \\ Qiulin Wanga Yonghe Hub Binghu $\mathrm{Li}^{\mathrm{c}}$ Xinzhong Hao ${ }^{\mathrm{d}}$ \\ aDepartment of Cardiology, The First Affiliated Hospital, Chengdu Medical College, Chengdu, \\ bDepartment of Integrated Traditional Chinese and Western Medicine, Chengdu Military General \\ Hospital,Chengdu, cDepartment of Neurology, Institute of Surgery Research, Daping Hospital, Third \\ Military Medical University, Chongqing, dDepartment of Cardiology, Nephrology and Endocrinology, \\ The 37th Hospital of Chinese People's Liberation Army, Ya'an, China
}

\section{Key Words}

Cinnamaldehyde • Diabetes • NF-E2-related factor $2 \cdot$ Endothelium • Oxidative stress

\begin{abstract}
Background/Aims: It is well documented that hyperglycemia-induced oxidative stress is an important causative factor of endothelial dysfunction. Cinnamaldehyde (CA) is a key flavor compound in cinnamon essential oil that can enhance the antioxidant defense against reactive oxygen species (ROS) by activating NF-E2-related factor 2 (Nrf2), which has been shown to have a cardiovascular protective effect, but its role in endothelial dysfunction induced by high glucose is unknown. Methods: Dissected male C57BL/6J mouse aortic rings and HUVECs were cultured in normal glucose(NG $5.5 \mathrm{mM}$ ) or high glucose(HG $30.0 \mathrm{mM})$ DMEM treatment with or without CA $(10 \mu \mathrm{M})$. Results: Treatment with CA protected the endothelium relaxation, inhibited ROS generation and preserved nitric oxide (NO) levels in the endothelium of mouse aortas treated with high glucose. CA up-regulated Nrf2 expression, promoted its translocation to the nucleus, and increased HO-1, NQO1, Catalase and Gpx1 expression under high glucose condition. The increased level of nitrotyrosine in HUVECs under high glucose was also attenuated by treatment with CA. Dihydroethidium (DHE) and DAF-2DA staining indicated that CA inhibited the ROS generation and preserved the NO levels in HUVECs, but these effects were reversed by Nrf2-siRNA in high glucose conditions. Conclusion: Our results indicated that CA protected endothelial dysfunction under high glucose conditions and this effect was mediated by Nrf2 activation and the up-regulation of downstream target proteins. CA administration may represent a promising intervention in diabetic patients who are at risk for vascular complications.
\end{abstract}

F. Wang and C. Pu contribute equally to this work.

\begin{tabular}{ll}
\hline $\begin{array}{l}\text { Peijian Wang } \\
\text { or Yonghe Hu }\end{array}$ & $\begin{array}{l}\text { Department of Cardiology, The First Affiliated Hospital, Chengdu Medical College, } \\
\text { Chengdu, Sichuan } 610500 \text { (PR China); or Department of Integrated Traditional } \\
\end{array}$ \\
& Chinese and Western Medicine, Chengdu Military General Hospital, Chengdu, Sichuan \\
& 610083 (PR China); E-Mail wpjmed@aliyun.com_or E-Mail huyonghe@vip.126.com
\end{tabular}




\section{Cellular Physiology Cell Physiol Biochem 2015;36:315-324 \\ \begin{tabular}{ll|l} 
and Biochemistry & $\begin{array}{l}\text { DOI: 10.1159/000374074 } \\
\text { Published online: May 05, } 2015\end{array}$ & $\begin{array}{l}\text { C } 2015 \mathrm{~S} . \text { Karger AG, Basel } \\
\text { www.karger.com/cpb }\end{array}$ \\
\cline { 2 - 3 } & Wang et al.: Cinnamaldehyde Prevents Endothelial Dysfunction Under High Glucose
\end{tabular}}

\section{Introduction}

Diabetes mellitus (DM) is a major and an increasing health problem worldwide. Its related vascular complications are the major cause of morbidity and mortality [1]. Studies have shown that markers of chronic, low-grade inflammation are correlated with markers of endothelial dysfunction in diabetic patients without clinical signs of macrovascular disease, such as ischemic heart disease and stroke [2]. It is well documented that the major cause of this inflammatory injury can be attributed to hyperglycemia-induced reactive oxygen species (ROS) generation [3]. Given the importance of oxidative stress in diabetic vascular complications, anti-oxidative stress treatments are thought to be an important intervention with the potential to ameliorate hyperglycemia-induced vascular lesions. However, the use of exogenous radical scavengers, such as vitamin $\mathrm{C}$ and vitamin $\mathrm{E}$, in large clinical trials remains controversial $[4,5]$.

The endogenous antioxidative enzyme NF-E2-related factor 2 (Nrf2) is thought to function as a physiological regulator of ROS generation and may contribute to the prevention of diabetes-related cardiovascular diseases [6]. Nrf2 regulates the expression of numerous genes, such as heme oxygenase $1(\mathrm{HO}-1)$ and $\mathrm{NAD}(\mathrm{P}) \mathrm{H}$ dehydrogenase, quinone 1 (NQO1), by interacting with antioxidant response elements in target gene promoters, and the protein products of these genes neutralize free radicals and accelerate the removal of environmental toxins [6].

Previous studies indicated that cinnamic aldehyde (CA), the key flavor compound in cinnamon essential oil extracted from Cinnamomum zeylanicum and Cinnamomum cassia bark, could reduce disease onset or improve prognosis [7]. CA enhanced the antioxidant defense against ROS produced under hyperglycemic conditions and thus protected pancreatic beta cells and exhibited anti-diabetic properties [8]; it also prevented the development of hypertension in insulin deficiency and insulin resistance by normalizing vascular contractility and exerting an insulinotropic effect [9]. Additionally, CA induces endothelium-dependent vasorelaxant activity in isolated rat aortas [10]. More importantly, CA is a potent Nrf2 inducer, and when used at low doses incapable of eliciting cytotoxicity, it may thus serve as a cancer chemopreventive agent [11]. CA activates the Nrf2-dependent antioxidant response in human epithelial colon cells [12]. In a streptozotocin-induced diabetes model, CA has an Nrf2-dependent effect on the antioxidant status of the rat kidney [7]. The above studies indicated that CA has beneficial effects for diabetes and related complications. However, the direct effects and underlying mechanism of CA on endothelium dysfunction induced by high glucose are unknown.

The present study was performed to test the hypothesis that CA attenuates high glucoseinduced endothelial dysfunction through an Nrf2-mediated antioxidant effect.

\section{Materials and Methods}

\section{Artery and endothelial cell culture}

Eight week old male C57BL/6J mouse (Model Animal Research Center, Nanjing University, Jiangsu, PR China) aortic rings were dissected in sterile PBS and incubated in Dulbecco's modified eagle medium (DMEM) supplemented with $10 \%$ fetal bovine serum (FBS, Gibco), $100 \mathrm{IU} / \mathrm{mL}$ penicillin and $100 \mu \mathrm{g} / \mathrm{mL}$ streptomycin. The high-glucose (HG: $30 \mathrm{mM}$ ) condition was achieved by the addition of $24.5 \mathrm{mM}$ D-glucose, and $24.5 \mathrm{mM}$ of mannitol was used in the normal glucose (NG) osmotic control condition [13]. After the 24-h incubation period, the aortic rings were transferred to a chamber filled with fresh Krebs solution and mounted in a wire myograph (DMT 620_M, Danmark) to measure changes in the isometric force. HUVECs (CHI Scientific, Inc, China) were grown in DMEM supplemented with 10\% FBS and 1\% antibiotics. Cultured aortic rings and cells were maintained at $37^{\circ} \mathrm{C}$ in a humidified atmosphere of $95 \%$ air $/ 5 \% \mathrm{CO}_{2}$. The cells were made quiescent by the incubation of $90 \%$ confluent cultures in serum-free DMEM and were then incubated with CA $(10 \mu \mathrm{M})$ for $24 \mathrm{~h}$ in the presence or absence of Nrf2 siRNA.

\section{KARGER}




\section{Cellular Physiology Cell Physiol Biochem 2015;36:315-324

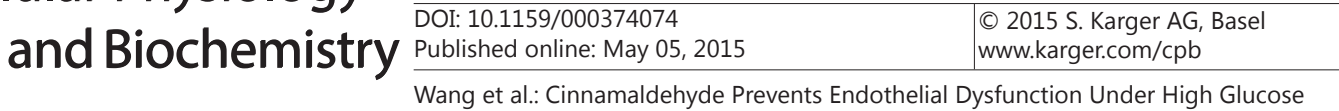

Cinnamaldehyde treatment

CA (Sigma-Aldrich, USA) diluted in DMSO was added to the incubation medium at the same time when the cells or artery treated by high glucose or normal glucose.The control group was treated by DMSO (final concentration was less than $0.1 \%$ ).

RNA interference of Nrf2

Small interfering RNAs (siRNAs) specific for Nrf2 were purchased from Santa Cruz Biotechnology, Inc. (USA), and the transfection of siRNAs was performed according to the manufacturer's instructions.

\section{Measurement of vascular activities}

Changes in the isometric tone of the aortic rings were recorded by wire myograph, as previously described [13]. The arterial segments were stretched to an optimal baseline tension $(2 \mathrm{mN})$ and then allowed to equilibrate for one hour before being contracted with $60 \mathrm{mM} \mathrm{KCl}$ and rinsed in Krebs solution. Endothelium-dependent relaxation was measured by testing the concentration-response relationship upon the cumulative addition of acetylcholine (ACh) to phenylephrine (Phe)-precontracted rings. The endothelium-independent relaxation response to nitroglycerine (NTG) was also measured in artery rings.

\section{Measurement of ROS production}

To assess superoxide production, dihydroethidium (DHE; Sigma-Aldrich, USA) staining was performed according to a previously described method. Aortic segments and cells were incubated with $40 \mu \mathrm{M}$ DHE for $45 \mathrm{~min}$ at $37^{\circ} \mathrm{C}$, after which the sections or cells were washed three times in DHE-free Krebs solution, the aortic sections were cut open, and the endothelium was inverted and placed between two coverslips for microscopic analysis [14]. Images were acquired, and the fluorescence intensity was analyzed.

\section{Evaluation of NO levels}

The NO levels in HUVECs and aortic segments were assessed by staining with diaminofluorescein-2 diacetate (DAF-2 DA, Sigma-Aldrich, USA) in Krebs solution for $45 \mathrm{~min}$ at $37^{\circ} \mathrm{C}$ followed by three washes with Krebs solution. Arteries were prepared as described above. The NO fluorescence was detected and the fluorescence intensity was analyzed as described above.

\section{Western blotting analysis}

Immunoblots of Nrf2, nitrotyrosine, NQO1, HO-1, Catalase(CAT), Glutathione peroxidase 1(GPx-1) and GAPDH were prepared as previously described [15]. After incubation with secondary antibodies (ZSGB-BIO, China) at room temperature for $2 \mathrm{~h}$, the proteins were detected with enhanced chemiluminescence and quantified using a Gel Doc 2000 Imager (Bio-Rad, USA). Protein expression was normalized to GAPDH. All of the primary antibodies were purchased from Santa Cruz Biotechnology (Santa Cruz, CA, USA).

\section{Statistical analysis}

Data are the means \pm SEM. The maximum response $(E \max )$ was calculated from individual agonist concentration-response curves using GraphPad Prism 3.0 (GraphPad Software, San Diego, CA). The significant differences in mean values were assessed by Student's $t$-test. Two-sided $P$ values $<0.05$ were considered statistically significant.

\section{Results}

Cinnamaldehyde preserved in vitro endothelium-dependent relaxation of mice aortas under high glucose

High-glucose (HG) exposure for $24 \mathrm{~h}$ impaired the ACh-induced endothelium-dependent relaxation of aortic rings compared with the normal-glucose (NG) exposure condition. The presence of CA $(10 \mu \mathrm{M})$ in high glucose preserved the endothelium-dependent relaxation(Fig. $1 \mathrm{~A})$. In normal glucose condition, CA improved ACh induced relaxation slightly, but there were no significant differences between the CA group and Control group, $P>0.05$ (Fig. 1A). The endothelium-independent relaxation induced by nitroglycerin was similar in each group (Fig. 1B). 


\section{Cellular Physiology Cell Physiol Biochem 2015;36:315-324

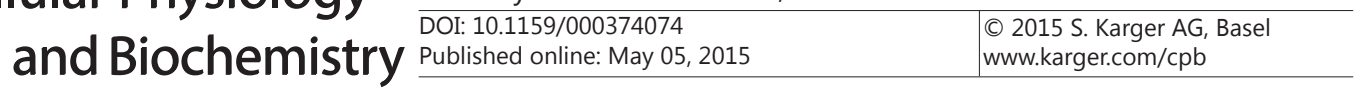

A

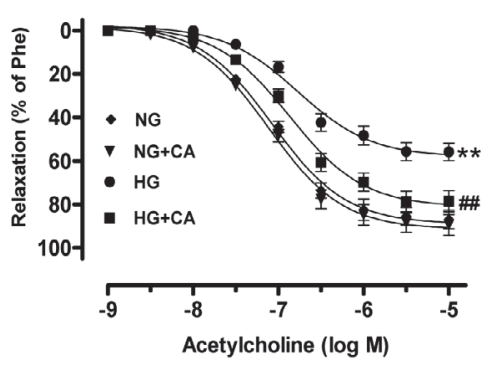

B

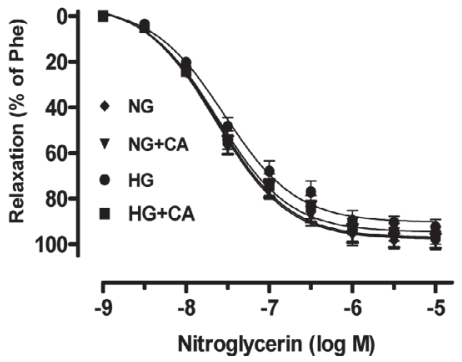

Fig. 1. Cinnamaldehyde preserved in vitro endothelium-dependent relaxation of mice aortas under high glucose conditions. (A) High-glucose exposure (HG, glucose $30 \_\mathrm{mM}, 24 \mathrm{~h}$ ) impaired the endothelium-dependent relaxation of mouse aortas in vitro. Cinnamaldehyde $(\mathrm{CA}, 10 \mu \mathrm{M})$ treatment ameliorated endothelial dysfunction in mouse aortas under high-glucose conditions but not in normal-glucose conditions. ${ }^{* *} P<0.01$ versus the NG group; ${ }^{\#} P<0.01$ versus the HG group. (B) Endothelium-independent relaxation is shown for all groups tested. Data are the means \pm SEM. In each case, $n=6$ samples from different mice.

Fig. 2. Cinnamaldehyde inhibited ROS production and preserved NO levels in mice aortas treated with high glucose ex vivo. (A and C) ROS production measured by DHE staining in the en face endothelium of aortas from C57BL/6J mice after treatment with NG (NG group), NG plus CA $(\mathrm{NG}+\mathrm{CA}, \quad 10$ $\mu \mathrm{M}), \mathrm{HG}$ (HG group) or HG plus CA (HG+CA, 10 $\mu \mathrm{M})$. (B and D) NO production measured by DAF-2DA staining in the

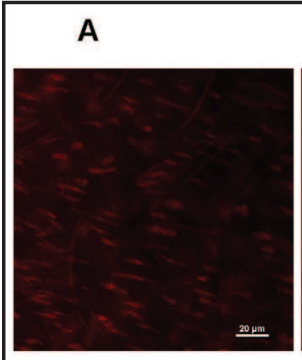

NG

B

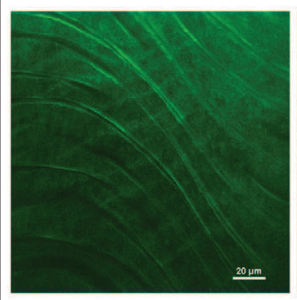

NG

C

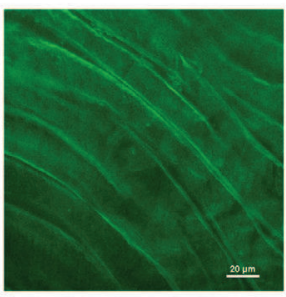

$N G+C A$

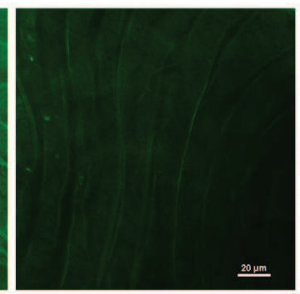

HG

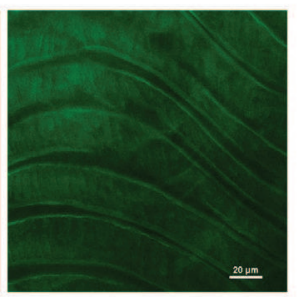

$H G+C A$

D

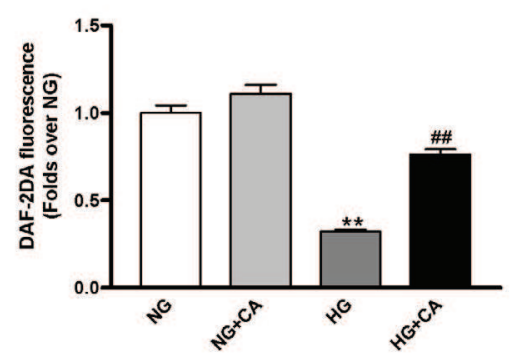

en face endothelium of aorta arteries. Red: DHE fluorescence. Green: DAF-2DA fluorescence. ${ }^{* *} P<0.01$ versus the NG group; ${ }^{\# \#} P<0.01$ versus the HG group. Data are the means \pm SEM. In each case, $\mathrm{n}=6$ samples from different mice. The scale bar indicates $20 \mu \mathrm{m}$.

\section{KARGER}




\section{Cellular Physiology Cell Physiol Biochem 2015;36:315-324 \begin{tabular}{l|l} 
and Biochemistry $\begin{array}{l}\text { DOt:1159/000374074 } \\
\text { Published online: May 05, 2015 }\end{array}$ & $\begin{array}{l}\text { O 2015 S. Karger AG, Basel } \\
\text { www.karger.com/cpb }\end{array}$ \\
\cline { 2 - 3 }
\end{tabular} \\ Wang et al.: Cinnamaldehyde Prevents Endothelial Dysfunction Under High Glucose}

Fig. 3. Cinnamaldehyde acts as an Nrf2 activator in high glucose-treated HUVECs. (A and B) Nrf2 expression(A) and localization(B) were assessed in HUVECs incubated in NG, HG, or $\mathrm{HG}+\mathrm{CA}$ DMEM media for $24 \mathrm{~h}$. Incubation in $\mathrm{HG}$ as well as treatment with $\mathrm{HG}+\mathrm{CA}$ increased Nrf2 staining intensity and translocation to the nucleus. ${ }^{* *} P<0.01$ versus the $N G$ group; ${ }^{\# \#} P<0.01$ versus the HG group. Data are the means \pm SEM. In each case, $\mathrm{n}=3$. The scale bar indicates $10 \mu \mathrm{m}$.

Cinnamaldehyde inhibited ROS production and prevented NO reduction in mouse aortas treated with high glucose in vitro

ROS production measured by DHE fluorescence in the en face endothelium of the aorta was higher in the HG than in the NG group. CA $(10 \mu \mathrm{M})$ treatment reduced ROS production in the endothelium of the aorta (Fig. 2). DAF-2DA staining indicated increased ROS levels in the en face endothelium of the aorta accompanied by decreased NO levels, but treatment with CA $(10 \mu \mathrm{M})$ preserved NO levels in the endothelium under high glucose conditions (Fig. 2A and 2B). Under normal glucose condition, CA treatment decreased the ROS levels and increased

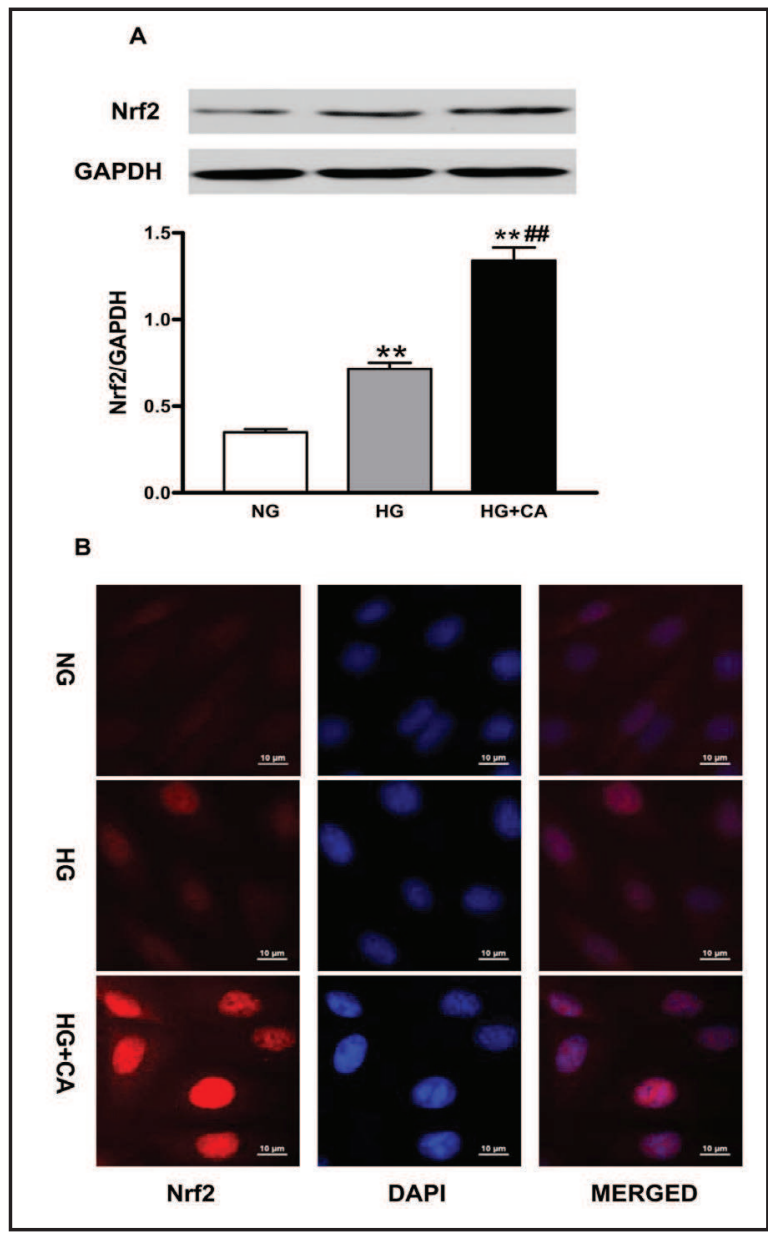
NO levels slightly, but there were no significantly difference between the NG+CA group and NG group, $P>0.05$.

\section{Cinnamaldehyde acts as an Nrf2 activator in high glucose-treated ECs}

Previous studies demonstrated that CA exerted anti-oxidative properties by activating Nrf2 in human skin and renal mesangial cells $[7,12]$, but whether CA has a similar effect on ECs under high glucose conditions was unknown. Western blotting indicated that the expression of Nrf2 was slightly increased under hyperglycemic conditions, and CA treatment further increased the Nrf2 levels. Immunofluorescent staining revealed predominant nuclear localization of Nrf2 in response to CA treatment. The above results indicated the activation of the Nrf2 pathway by CA treatment in ECs exposed to high glucose. We then investigated whether the induction of the Nrf2 pathway by CA could also affect the Nrf2 downstream genes, such as HO-1, NQ01, CAT and GPX-1. As shown in Fig. 4A-D, HO-1, NQO1, CAT and GPX1 expression under high glucose condition were increased by CA. The interaction between $\mathrm{O}_{2}$ and nitric oxide (NO) forms reactive peroxynitrite (ONOO-) [16]. We then asked whether the activation of Nrf2 could prevent tyrosine nitration in ECs treated with high glucose. Immunoblotting showed that the level of nitrotyrosine in the CA treatment group was lower than in the HG group (Fig. 4E).

Cinnamaldehyde diminished ROS levels and preserved NO levels in ECs under high glucose conditions

To determine the relationship between CA and the Nrf2 signal pathway, Nrf2 siRNA was used. We first confirmed that Nrf2 siRNA significantly blocked Nrf2 expression in high glucose-treated ECs, but control siRNA did not (Fig. 5A). We next investigated whether 


\section{Cellular Physiology Cell Physiol Biochem 2015;36:315-324

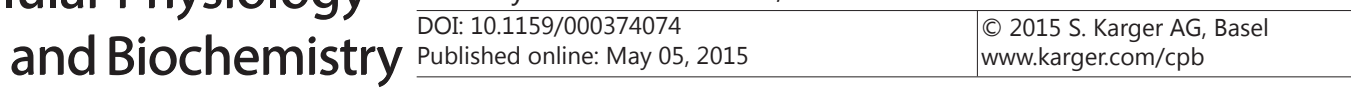 \\ Wang et al.: Cinnamaldehyde Prevents Endothelial Dysfunction Under High Glucose}

Fig. 4. Effects of Cinnamaldehyde on protein expression of HO-1, NQ01, CAT, GPX-1 and levels of nitrotyrosine. (A, B, C and D) Effects of $\mathrm{NG}$, high-glucose (HG, glucose $30 \mathrm{mM}$ ) and $\mathrm{HG}$ plus CA on HO-1, NQ01, CAT and GPX-1 expression levels detected by Western blotting. ${ }^{*} P<0.01$ versus the normal glucose group (NG, glucose $5.5 \mathrm{mM}) ;{ }^{\# \#} P<0.01$ versus the HG group. (E) Effect of CA treatment on the nitrotyrosine levels in HUVECs observed by Western blotting. ${ }^{* *} P<0.01$ versus the NG group; ${ }^{\#} P<0.01$ versus $\mathrm{HG}$ group. Data are the means \pm SEM. In each case, $\mathrm{n}=3$.
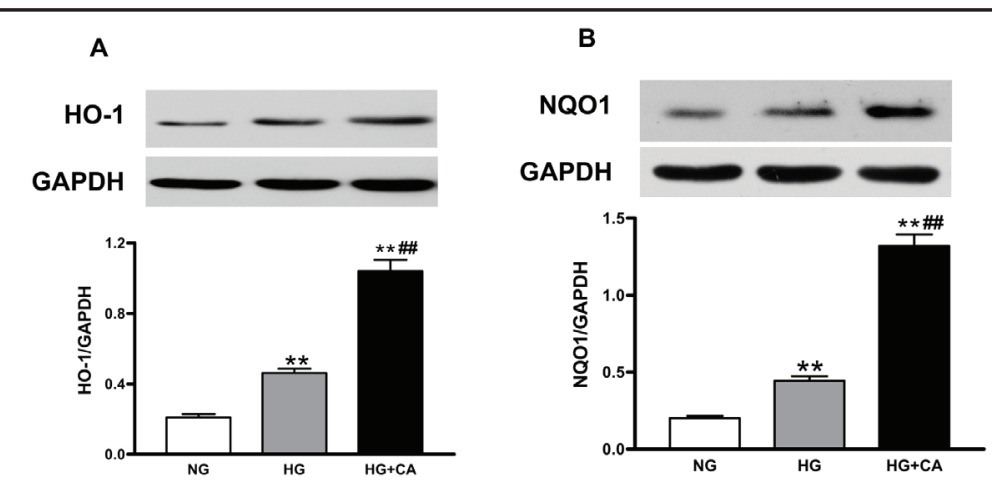

C
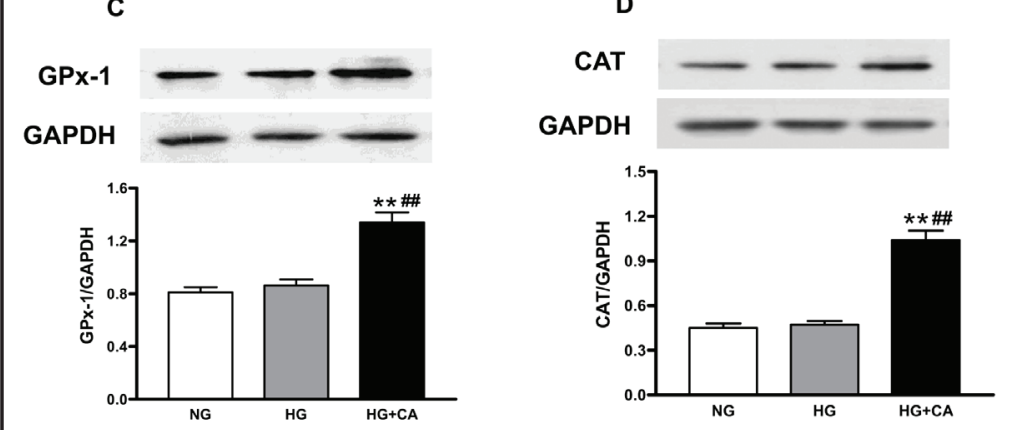

E
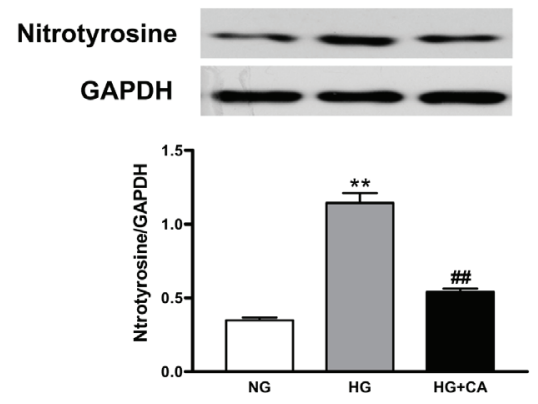

siRNA of Nrf2 could affect the downstream targets, western blotting results indicated the increased levels of HO-1 and NQO1 by CA were all reversed by siRNA of Nrf2 under high glucose condition (Fig. 5B and C). High glucose increased the ROS levels compared with NG $(5.5 \mathrm{mM})$. Treatment with CA $(10 \mu \mathrm{M})$ diminished the ROS levels, and this effect was antagonized by Nrf2 signal interference (Fig. 5D and F). We then asked whether this effect would preserve the NO levels under hyperglycemic conditions. DAF-2DA staining indicated that CA treatment prevented the decrease of NO levels under high glucose conditions, and this effect was also blocked by Nrf2 inhibition (Fig. 5E and G). These results indicated that the CA-mediated diminution of ROS production and preservation of NO levels in ECs under high glucose is associated with Nrf2 signaling.

\section{Discussion}

In the present study, we have shown for the first time that treatment with CA prevented high glucose-induced ROS generation and preserved NO levels in the endothelium, thus preserving the endothelium-dependent relaxation but not the endothelium-independent relaxation. Furthermore, CA up-regulated Nrf2 expression and promoted its nuclear 


\section{Cellular Physiology Cell Physiol Biochem 2015;36:315-324

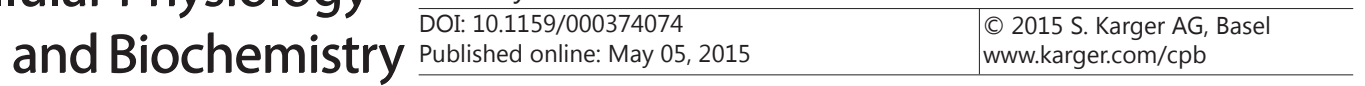

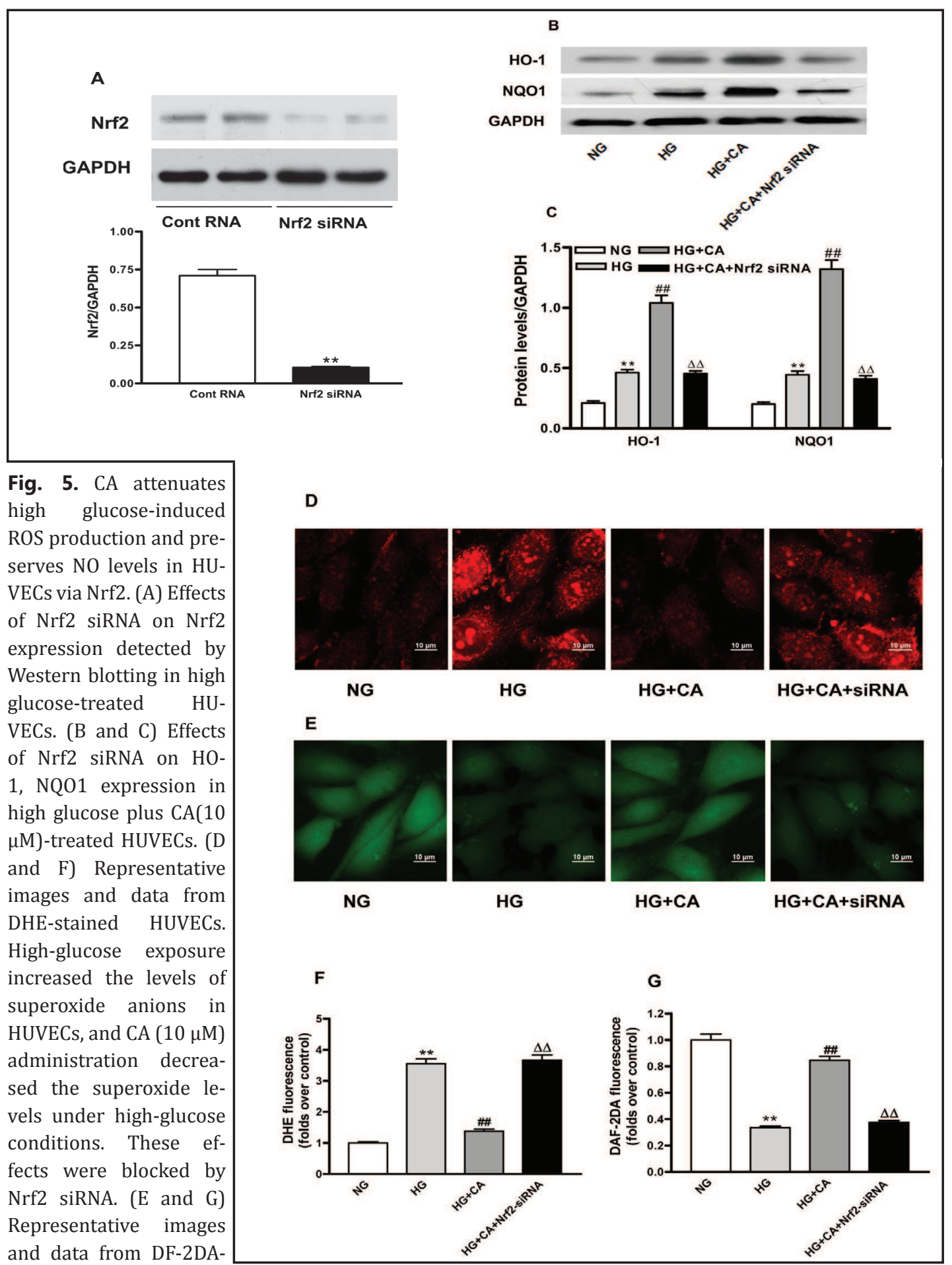

and data from DF-2DAstained HUVECs. High-glucose exposure decreased the level of NO in HUVECs, and CA increased the NO level under high-glucose conditions. These effects were blocked by Nrf2 siRNA. ${ }^{* *} P<0.01$ versus the NG group; ${ }^{\# \#} P$ $<0.01$ versus the HG group; ${ }^{\triangle} P<<0.01$ versus the $H G+C A$ group. Data are the means \pm SEM from 4 independent experiments. The scale bar indicates $10 \mu \mathrm{m}$.

translocation, inducing the expression of target proteins HO-1, NQO1, CAT and GPx-1 in high glucose-treated HUVECs and thus also decreasing the level of nitrotyrosine. In high glucosetreated HUVECs, CA treatment attenuated ROS generation and prevented NO depletion, but 


\section{Cellular Physiology Cell Physiol Biochem 2015;36:315-324

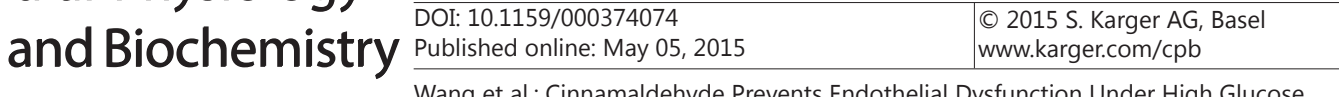

these effects were blocked by Nrf2 siRNA, indicating that CA prevented high glucose-induced endothelial dysfunction through endogenous Nrf2 signal activation.

Diabetes is characterized by hyperglycemia and the development of diabetic vascular complications. The primary causative factor leading to the pathophysiologic alterations in the diabetic vasculature is exposure to a high blood glucose level [17]. In spite of the significant developments in anti-diabetic therapy, diabetic vascular complications continue to be seriously detrimental [17]. Therefore, it is critical to pursue novel strategies for preventing the vascular complications associated with diabetes.

CA is a spice compound in cinnamon that has been widely used as a component in perfumes, as a fungicide, and as a flavoring agent in foodstuffs, such as chewing gum, ice cream, candy and beverages [18]. Increasing evidence has demonstrated that CA has many pharmacological activities, including anti-hyperglycemic, anti-oxidative stress, anti-cancer, cardiovascular protective, etc. $[9,19,20]$, indicating that CA may play a protective role in diabetic vascular complications, but its direct effect on endothelial dysfunction induced by high glucose was unknown.

The accelerated degradation of NO by ROS is most likely the major mechanism that limits NO bioavailability in states of cardiovascular disease [21]. In our study, we first found that the endothelial ROS level increased and the NO level decreased after high glucose treatment for 24_h; these changes may be responsible for the impairment of endotheliumdependent relaxation. Pretreatment with CA attenuates the ROS generation and prevented the decrease of NO, thus preserving endothelium-dependent relaxation in mice aortas under hyperglycemic conditions. These results showed the antioxidant properties and endothelial protective effects of CA under high glucose conditions.

We next asked how CA protected the endothelium under hyperglycemic conditions. Previous studies indicated that dietary cinnamon-derived CA activates the Nrf2dependent antioxidant response in human epithelial colon cells and may therefore represent a chemopreventive dietary factor targeting colorectal carcinogenesis [22]. CA enhances Nrf2 nuclear translocation to upregulate phase II detoxifying enzyme expression in HepG2 cells [11]. These studies indicate that CA is an Nrf2 activator. However, whether CA also acts as an Nrf2 activator in the endothelium under hyperglycemia is unknown. In our study, Western blotting indicated that CA increased Nrf2 expression and promoted Nrf2 nuclear translocation in HUVECs exposed to high glucose. These results indicated that CA also acts as an Nrf2 activator in high glucose-treated HUVECs.

Nrf2, first cloned and characterized by its ability to bind to the NF-E2/AP-1 repeat in the promoter of the beta-globin gene, is ubiquitously expressed in many organs as a transcriptional activator of phase II detoxifying genes by binding to antioxidant responsive element (ARE) sequences [23].

Many cardiovascular diseases, including diabetic vascular complications, are associated with a failure of defenses against oxidative stress-induced cellular damage and/or death, leading to organ dysfunction [24]. It is well documented that Nrf2 plays a protective role in cardiovascular diseases such as atherosclerosis, hypertension, and heart failure due to its antioxidant properties [25]. Nrf2 activation depends on Kelch ECH Associating Protein 1 , a cytoskeletal protein that binds to actin filaments and Nrf2 to prevent the nuclear translocation of Nrf2, thus acting as a transcriptional repressor during basal conditions [25].

HO-1 is well known for its cytoprotective effects against oxidative injuries and inflammation in vitro and in vivo [26]. The overexpression of HO-1 plays a protective role in myocardial ischemia/reperfusion injury in diabetic mice [27]. The treatment of endothelial cells with high glucose for 7 days decreased the HO-1 activity and cell viability [28]. HO-1 up-regulation in diabetic rats led to reduced ROS production and decreased endothelial cell sloughing [29]. It has been suggested that inducible NQ01, which is highly and constitutively expressed in cardiovascular cells, may act as a superoxide scavenger [30]. Thus, the upregulation of HO-1 and NQO1 in response to high glucose provides an effective endogenous antioxidant defense mechanism in diabetes and other vascular diseases [6]. We next explored whether CA could further increase the expression of the downstream 


\section{Cellular Physiology Cell Physiol Biochem 2015;36:315-324

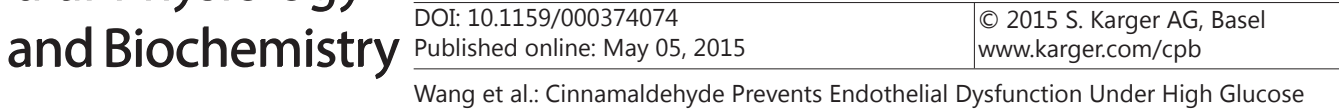

proteins HO-1 and NQ01. Immunoblotting confirmed that CA increased both HO-1, NQO1, CAT and GPx-1 expression in HUVECs under high glucose conditions, and these effects may be responsible for the decreased nitrotyrosine levels.

Finally, we investigated whether the effects of CA on endothelial cells was associated with Nrf2. DHE and DAF-2DA staining demonstrated that CA has direct effects on ROS and NO levels in ECs under high glucose, and these effects were blocked by Nrf2 siRNA. These results confirmed that the induction of ROS generation by CA under high glucose conditions was mediated by Nrf2 signal pathway activation.

In summary, this study shows that treatment with CA can prevent high glucose-induced endothelial dysfunction. Our mechanistic evidence suggests that this vascular benefit is likely to result from an enhancement of $\mathrm{Nrf} 2$ expression and nuclear translocation, which leads to the up-regulation of both HO-1, NQ01, CAT and GPx-1 expression and attenuates the generation of ROS and nitrotyrosine levels in ECs under high glucose conditions. Our findings provide new insight into the direct role of CA in diabetes vascular dysfunction. Nrf2 activation by CA may represent a promising intervention in diabetic patients who are at risk for vascular complications.

\section{Acknowledgments}

This research were supported by the National Natural Science Foundation of China (81400289), grants from the Scientific Research Fund of Chengdu Medical College (CYZ13001 ) and by Scientific Research Fund of SiChuan Provincial Education Department (14ZB0234).

\section{Disclosure Statement}

The authors declare that there is no conflict of interests regarding the publication of this paper.

\section{References}

1 van Dieren S, Beulens JW, van der Schouw YT, Grobbee DE, Neal B: The global burden of diabetes and its complications: an emerging pandemic. Eur J Cardiovasc Prev Rehabil 2010;17:S3-8.

2 Omar EA, Kam A, Alqahtani A, Li KM, Razmovski-Naumovski V, Nammi S, Chan K, Roufogalis BD, Li GQ: Herbal medicines and nutraceuticals for diabetic vascular complications: mechanisms of action and bioactive phytochemicals. Curr Pharm Des 2010;16:3776-3807.

3 Orasanu G, Plutzky J: The pathologic continuum of diabetic vascular disease. J Am Coll Cardiol 2009;53:S35-42.

4 Lonn E, Bosch J, Yusuf S, Sheridan P, Pogue J, Arnold JM, Ross C, Arnold A, Sleight P, Probstfield J, Dagenais GR; HOPE and HOPE-TOO Trial Investigators: Effects of long-term vitamin E supplementation on cardiovascular events and cancer: a randomized controlled trial. JAMA 2005;293:1338-1347.

5 Sesso HD, Buring JE, Christen WG, Kurth T, Belanger C, MacFadyen J, Bubes V, Manson JE, Glynn RJ, Gaziano JM: Vitamins E and C in the prevention of cardiovascular disease in men: the Physicians' Health Study II randomized controlled trial. JAMA 2008;300:2123-2133.

6 He M, Siow RC, Sugden D, Gao L, Cheng X, Mann GE: Induction of HO-1 and redox signaling in endothelial cells by advanced glycation end products: a role for Nrf2 in vascular protection in diabetes. Nutr Metab Cardiovasc Dis 2011;21:277-285.

7 Zheng H, Whitman SA, Wu W, Wondrak GT, Wong PK, Fang D, Zhang DD: Therapeutic potential of Nrf2 activators in streptozotocin-induced diabetic nephropathy. Diabetes 2011;60:3055-3066.

8 Subash-Babu P, Alshatwi AA, Ignacimuthu S: Beneficial Antioxidative and Antiperoxidative Effect of Cinnamaldehyde Protect Streptozotocin-Induced Pancreatic beta-Cells Damage in Wistar Rats. Biomol Ther (Seoul) 2014;22:47-54. 


\section{Cellular Physiology Cell Physiol Biochem 2015;36:315-324

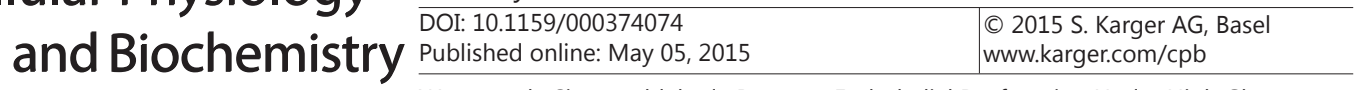 \\ Wang et al.: Cinnamaldehyde Prevents Endothelial Dysfunction Under High Glucose}

9 El-Bassossy HM, Fahmy A, Badawy D: Cinnamaldehyde protects from the hypertension associated with diabetes. Food Chem Toxicol 2011;49:3007-3012.

10 Yanaga A, Goto H, Nakagawa T, Hikiami H, Shibahara N, Shimada Y: Cinnamaldehyde induces endotheliumdependent and -independent vasorelaxant action on isolated rat aorta. Biol Pharm Bull 2006;29:24152418.

11 Huang TC, Chung YL, Wu ML, Chuang SM: Cinnamaldehyde enhances Nrf2 nuclear translocation to upregulate phase II detoxifying enzyme expression in HepG2 cells. J Agric Food Chem 2011;59:5164-5171.

12 Wondrak GT, Cabello CM, Villeneuve NF, Zhang S, Ley S, Li Y, Sun Z, Zhang DD: Cinnamoyl-based Nrf2activators targeting human skin cell photo-oxidative stress. Free Radic Biol Med 2008;45:385-395.

13 Sun J, Pu Y, Wang P, Chen S, Zhao Y, Liu C, Shang Q, Zhu Z, Liu D: TRPV1-mediated UCP2 upregulation ameliorates hyperglycemia-induced endothelial dysfunction. Cardiovasc Diabetol2013;12:69.

14 Tian XY, Wong WT, Xu A, Lu Y, Zhang Y, Wang L, Cheang WS, Wang Y, Yao X, Huang Y: Uncoupling protein-2 protects endothelial function in diet-induced obese mice. Circ Res 2012;110:1211-1216.

15 Wang P, Yan Z, Zhong J, Chen J, Ni Y, Li L, Ma L, Zhao Z, Liu D, Zhu Z: Transient receptor potential vanilloid 1 activation enhances gut glucagon-like peptide- 1 secretion and improves glucose homeostasis. Diabetes 2012;61:2155-2165.

16 Munzel T, Heitzer T, Harrison DG: The physiology and pathophysiology of the nitric oxide/superoxide system. Herz 1997;22:158-172.

17 Sasaki S, Inoguchi T: The role of oxidative stress in the pathogenesis of diabetic vascular complications. Diabetes Metab J 2012;36:255-261.

18 Youn HS, Lee JK, Choi YJ, Saitoh SI, Miyake K, Hwang DH, Lee JY: Cinnamaldehyde suppresses toll-like receptor 4 activation mediated through the inhibition of receptor oligomerization. Biochem Pharmacol 2008;75:494-502.

19 Subash Babu P, Prabuseenivasan S, Ignacimuthu S: Cinnamaldehyde--a potential antidiabetic agent. Phytomedicine 2007;14:15-22.

20 Zhang Y, Cao W, Xie YH, Yang Q, Li XQ Liu XX, Wang SW: The comparison of alpha-bromo-4chlorocinnamaldehyde and cinnamaldehyde on coxsackie virus B3-induced myocarditis and their mechanisms. Int Immunopharmacol 2012;14:107-113.

21 Hare JM, Stamler JS: NO/redox disequilibrium in the failing heart and cardiovascular system. J Clin Invest 2005;115:509-517.

22 Wondrak GT, Villeneuve NF, Lamore SD, Bause AS, Jiang T, Zhang DD: The cinnamon-derived dietary factor cinnamic aldehyde activates the Nrf2-dependent antioxidant response in human epithelial colon cells. Molecules 2010;15:3338-3355.

23 Lau A, Villeneuve NF, Sun Z, Wong PK, Zhang DD: Dual roles of Nrf2 in cancer. Pharmacol Res 2008;58:262270.

24 Maksimenko AV, Vavaev AV: Antioxidant enzymes as potential targets in cardioprotection and treatment of cardiovascular diseases. Enzyme antioxidants: the next stage of pharmacological counterwork to the oxidative stress. Heart Int 2012; 7:e3.

25 Howden R: Nrf2 and cardiovascular defense. Oxid Med Cell Longev 2013:104308.

26 Chauveau C, Rémy S, Royer PJ, Hill M, Tanguy-Royer S, Hubert FX, Tesson L, Brion R, Beriou G, Gregoire M, Josien R, Cuturi MC, Anegon I: Heme oxygenase-1 expression inhibits dendritic cell maturation and proinflammatory function but conserves IL-10 expression. Blood 2005;106:1694-1702.

27 Liu X, Wei J, Peng DH, Layne MD, Yet SF: Absence of heme oxygenase-1 exacerbates myocardial ischemia/ reperfusion injury in diabetic mice. Diabetes 2005;54:778-784.

28 Abraham NG, Kushida T, McClung J, Weiss M, Quan S, Lafaro R, Darzynkiewicz Z, Wolin M: Heme oxygenase- 1 attenuates glucose-mediated cell growth arrest and apoptosis in human microvessel endothelial cells. Circ Res 2003;93:507-514.

29 Peterson SJ, Frishman WH, Abraham NG: Targeting heme oxygenase: therapeutic implications for diseases of the cardiovascular system. Cardiol Rev 2009;17:99-111.

30 Siegel D, Gustafson DL, Dehn DL, Han JY, Boonchoong P, Berliner LJ, Ross D: NAD(P)H:quinone oxidoreductase 1: role as a superoxide scavenger. Mol Pharmacol 2004;65:1238-1247. 